\title{
Poems Syndrome with Vitiligo: Complete Remission and Repigmentation Post Autologous Stem Cell Transplant
}

\author{
Raheel Iftikhar*, Syed Kamran Mahmood, Tariq \\ Mehmood Satti and Qamar un nisa Chaudhry \\ Armed Forces Bone Marrow Transplant Centre, Pakistan \\ *Correspondling author: Raheel Iftikhar, Armed \\ Forces Bone Marrow Transplant Centre, Rawalpindi, \\ Pakistan
}

Received: J une 12, 2017; Accepted: July 03, 2017;

Published: July 24, 2017

\begin{abstract}
The acronym POEMS (polyneuropathy, organomegaly, endocrinopathy, monoclonal gammopathy, skin changes) represents a rare paraneoplastic syndrome due to underlying plasma cell disorder. Due to myriad non-specific presentation, most of these cases are missed or diagnosis is significantly delayed. The importance of early correct diagnosis is enormous as POEMS syndrome carries a significantly better prognosis than most of the other plasma cell disorders. Vitiligo is not a known skin manifestation of POEMS syndrome and often cosmetically severely disfiguring and very difficult to treat.

We are reporting case of a middle age male presenting with POEMS syndrome and vitiligo who underwent autologous stem cell transplant and achieved complete remission of POEMS syndrome and had skin repigmentation post hematopoietic stem cell transplant (HSCT).

Keywords: Polyneuropathy, Monoclonal gammopathy, Vitiligo, Stem cell transplantation
\end{abstract}

\section{Introduction}

POEMS syndrome also known as osteosclerotic myeloma, Crowfukase syndrome or Takatsuki syndrome is a rare monoclonal plasma cell disorder. Most of the clinical manifestations of this syndrome are remembered by acronym "POEMS" although it inadequately covers many defining features of this myriad syndrome [1]. Due to rarity of the syndrome and multisystem involvement, diagnosis is often delayed. Exact pathogenesis is not well known and is believed to be due to chronic over production of pro-inflammatory cytokines particularly vascular endothelial growth factor (VEGF). Risk adapted therapy, use of proteasome inhibitors and HSCT has resulted in dramatic improvement in long term survival of patients suffering from this disease [2]. Although patients with POEMS syndrome have many skin manifestations, vitiligo is not a documented cutaneous manifestation. We hereby present a case of POEMS syndrome and vitiligo in which disease remission post HSCT was also associated with regimentation of skin and resolution of vitiligo. To our knowledge, this is first case report highlighting this aspect in POEMS syndrome.

\section{Case Presentation}

A 46 years old male presented in outpatient department with 2 years history of off and on numbness of hands and feet, facial twitching and abdominal pain. With these complaints he was reviewed by endocrinologist and was found to have primary hypoparathyroidism and was started on calcium and vitamin-D replacement. He was investigated and no cause of hypoparathyroidism could be found. Two months late he had an episode of severe abdominal pain and was operated for appendicitis but histopathology of tissue showed normal non-inflamed appendix. His abdominal pain persisted and he started having low backache and numbness of hands and feet which were attributed initially to hypoparathyroidism, however numbness of hands and feet progressed and he developed glove and stocking peripheral neuropathy. He also noted appearance of hypopigmented skin patches over face and arms which progressed to involve trunk and back and was associated with excessive hair growth all over body. He was reviewed by dermatologist and advised narrow band ultraviolet $\mathrm{B}$ therapy and topical hydroquinone for vitiligo, however there was no improvement. His back pain worsened gradually and he also started having pain in left hip joint with antalgic gait. His blood complete picture done as a part of routine screening showed raised hemoglobin and platelet count and was referred to our institute for evaluation. On examination he had vitiligo, hyperpigmentation, hypertrichosis and white nails. Systemic examination revealed hepatosplenomegaly and glove and stocking peripheral neuropathy. Lab investigations revealed hemoglobin of $16.7 \mathrm{~g} / \mathrm{dl}$, hematocrit $49 \%$, platelet counts of $560,000 / \mathrm{ul}$. Bone marrow aspiration and trephine biopsy showed 6\% plasma cells which were CD38 and CD138 positive with increased megakaryocytes. PCR for JAK-2 mutation was negative. Serum protein electrophoresis showed a faint band in gamma region. His serum free light chains showed increase in lambda light chain with kappa: lambda of 4.2 (normal 0.6-1.65) and serum immunofixation showed IgG lambda. His Contrast enhanced computed tomography scans showed hepatosplenomegaly, lytic lesion left femur and mixed sclerotic and lytic lesions involving dorsolumbar spine (Figure 1A). Biopsy of vertebral sclerotic lesions was done and was suggestive of plasmacytoma. EMG/NCS showed sensorimotor distal axonal neuropathy involving all 4 limbs. His endocrine profile was repeated which showed primary hypoparathyroidism and hypogonadotrophic hypogonadism. His PET scan showed hypermetabolic skeletal, pulmonary and adrenal signals (Figure 1B). During admission he developed an episode of slurring of speech and generalized tonic clonic seizure. MRI brain was done, which showed acute parietooccipital infarct.

On the basis of polyneuropathy, skin changes, organomegaly,
Ann Hematol Oncol - Volume 4 Issue 8 - 2017

ISSN : 2375-7965 | www.austinpublishinggroup.com

Iftikhar et al. (C) All rights are reserved
Citation: Iftikhar R, Mahmood SK, Satti TM and Qamar un nisa Chaudhry. Poems Syndrome with Vitiligo: Complete Remission and Repigmentation Post Autologous Stem Cell Transplant. Ann Hematol Oncol. 2017; 4(8): 1165. 


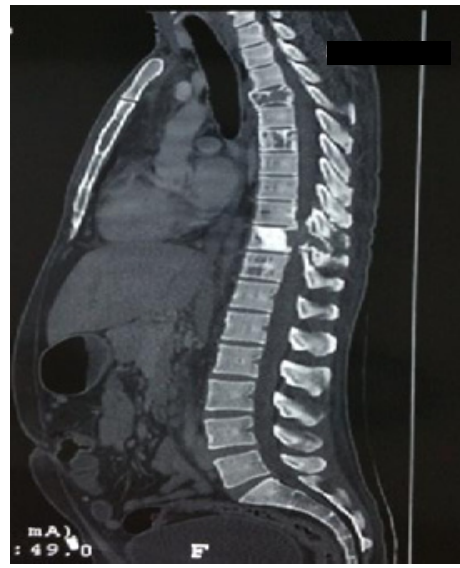

Figure 1A: Mixed sclerotic and lytic lesions involving dorsolumbar spine.

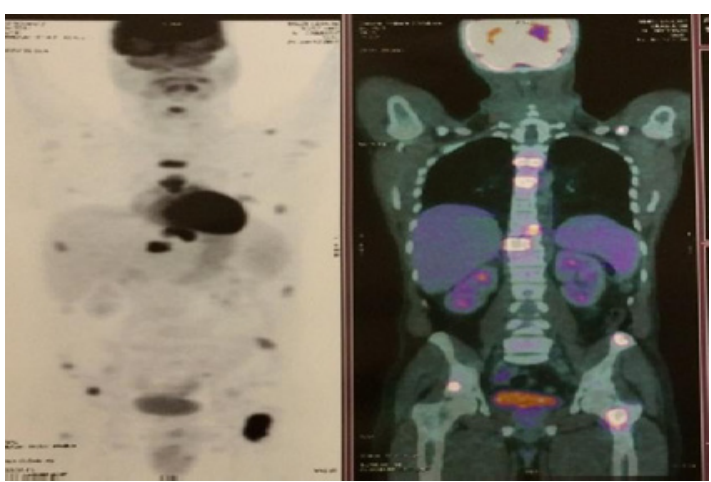

Figure 1B: PET-CT showing hypermetabolic skeletal, pulmonary and adrenal FDG avid signals.

endocrinopathy and monoclonal gammopathy, patient was diagnosed as having POEMS syndrome. He was started on cyclophosphamide, borezomib and dexamethasone (CyBorD) induction and was given 4 cycles of CyBorD. He had hematological and clinical complete remission after 4 cycles and consolidation was done with autologous stem cell transplantation. He was given cyclo-G mobilization and peripheral blood stem cell apheresis was done collecting $3.2 \times 10^{6} \mathrm{CD} 34$

Table 1: Diagnostic criteria of POEMS syndrome.

\begin{tabular}{|l|l|}
\hline Mandatory criteria & Polyneuropathy \\
\hline Major criteria & Monoclonal plasma cell disorder \\
\hline & Sclerotic or mixed sclerotic/lytic bone lesions \\
\hline & Castleman disease \\
\hline Minor criteria & Elevated serum or plasma vascular endothelial growth factor (VEGF) levels \\
\hline & Organomegaly (splenomegaly, hepatomegaly, or lymphadenopathy) \\
\hline & Edema (edema, pleural effusion, or ascites) \\
\hline & Endocrinopathy (adrenal, thyroid, pituitary, gonadal, parathyroid, pancreatic) \\
\hline Known Associations & Skin changes (hyperpigmentation, hypertrichosis, plethora, hemangiomata, white nails) \\
\hline Possible associations & Papilledema \\
\hline Diagnosis requires both major and atleast 1 minor criteria \\
\hline
\end{tabular}

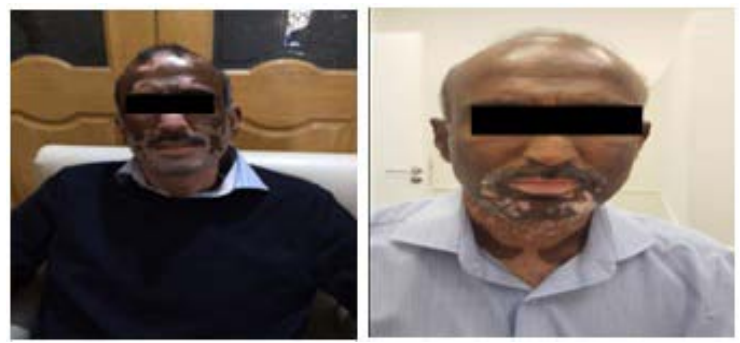

Figure 2: Regimentation of skin and normalization of hypertrichosis after Auto-HSCT.

cells. Melphalan $200 \mathrm{mg} / \mathrm{m}^{2}$ was used as myeloablative conditioning. He tolerated autologous transplant well except for CMV reactivation which was managed with valgancyclovir. Interestingly his hypertrichosis started settling 3 months post autologous transplant and his skin repigmentation started in patches of vitiligo (Figure 2). At present he is 6 months post-transplant, in CR and without any active issue.

\section{Discussion}

The acronym POEMS was coined by Bardwick in 1980. Although used widely it does not cover many important features of this unique disorder like papilledema, extravascular volume overload, thrombocytosis/erythrocytosis, thrombotic episodes, sclerotic bone lesions, elevated VEGF levels and Castleman variant. Initial reports came from Japan but later documented worldwide. As with other plasma cell dyscrasias, it commonly presents in the fifth to sixth decade. In a Mayo Clinic series of 99 patients, the median age was 51 years (range: 30 to 83 ) and 63 percent were males [3].

Exact pathogenesis is not known, VEGF is thought to play a role in disease symptomatology and progression. It role as primary trigger is not validated yet because of mixed results with anti-VEGF therapy. A composite clinical and laboratory criteria has been developed for diagnosis of POEMS syndrome. The IMWG (international myeloma working group) criteria for the diagnosis of POEMS syndrome require the presence of both mandatory, 1 major and atleast 1 minor criteria for diagnosis (Table 1) [4]. In addition a number of clinical features are seen which are currently not part of diagnostic criteria 
but reflect underlying pathobiologic basis of this unique disorder. Interesting to note is that despite multiorgan involvement, POEMS syndrome carries one of the best long term prognosis among plasma cell dyscrasias. Because of multisystem involvement diagnosis is often delayed and frequently overlooked and require clinical correlation to establish diagnosis and differentiate from monoclonal gammopathy of undetermined significance (MGUS), smoldering myeloma, light chain amyloid neuropathy, multiple myeloma.

Virtually any system can be affected in POEMS syndrome, so detailed history and thorough clinical examination is the single most initial step in evaluating these patients. Peripheral, sensorimotor, ascending neuropathy is seen in virtually all patients, papilledema is present in one third cases.

Skin manifestations include hypertrichosis, hyperpigmentation, white nails, acrocyanosis, hemangiomas and skin thickening [5]. Vitiligo is not a recognized manifestation of POEMS syndrome and its presence in our patient can be a co-existing finding or may represent a rare cutaneous manifestation of POEMS syndrome considering its resolution after induction chemotherapy and autologous HSCT in our patient. There is increased risk of arterial and venous thrombosis with $20 \%$ of patients experiencing documented thrombotic episode. In our patient there was episode of slurring of speech and generalized tonic clonic seizure and MRI brain showed acute parieto-occcipital infarction. Unlike other plasma cell dyscrasias, anemia is not a feature of POEMS syndrome, rather there is polycythemia and thrombocytosis as documented in our patient as well. Clonal plasma cells are present in only $20-25 \%$ patients on bone marrow examination and most of these are lambda positive [6]. VEGF levels are markedly elevated and correlate with disease activity. Extravascular fluid overload presents as pleural effusion, pericardial effusion and ascitesand is associated with unfavourable outcome. Endocrinopathy is a common (present in 85-90\%), important and frequently overlooked complication. Hypogonadismis most commonly seen followed by thyroid and parathyroid hormone abnormalities, disorders of glucose metabolism and adrenal involvement. Osteosclerotic, osteolytic and both sclerotic and lytic lesions can be present. Castleman's disease is a rare lymphoproliferative disorder with wide range of symptoms and presentations ranging from unifocal to multifocal and can be divided into plasma cell and hyaline variants. Castleman's disease and POEMS syndrome are frequently associated and approximately 15 percent of patients in the Mayo Clinic series with POEMS syndrome also had Castleman's disease.
Because of rarity of the syndrome and lack of clear insights in the pathogenesis, treatment is not standardized and ranges drom radiation therapy for isolated lesions to Bortezomib based triplet therapy and autologous HSCT. Melphalan is used as conditioning in doses ranging from $140-200 \mathrm{mg} / \mathrm{m}^{2}$. Of the 59 patients with POEMS syndrome treated at the Mayo Clinic Rochester, progression free survival was 98,94 , and $75 \%$ at 1,2 , and 5 years, respectively [7]. Vitiligo is not a documented cutaneous manifestation and its resolution post HSCT in our patient needs further research of possible pathogenetic mechanisms involved in resolution of vitiligo post HSCT.

To conclude POEMS syndrome is a rare, yet important paraneoplastic plasma cell disorder associated with multisystem involvement and carries a significantly favorable prognosis with current treatment approaches. Detailed history and examination, integrated clinical approach with appropriate investigations is important in reaching the diagnosis.

\section{References}

1. Dispenzieri A, Kyle RA, Lacy MQ, Rajkumar SV, Therneau TM, Larson DR, et al. POEMS syndrome: definitions and long-term outcome. Blood. 2003; 101: $2496-2506$

2. Li J, Zhou DB. New advances in the diagnosis and treatment of POEMS syndrome. Br J Haematol. 2013; 161: 303-315.

3. Kourelis TV, Buadi FK, Kumar SK, Gertz MA, Lacy MQ, Dingli D, et al. Longterm outcome of patients with POEMS syndrome: An update of the Mayo Clinic experience. Am J Hematol. 2016; 91: 585-589.

4. Dispenzieri A. POEMS syndrome: 2014 update on diagnosis, riskstratification, and management. Am J Hematol. 2014; 89: 214-223.

5. Dispenzieri A. POEMS Syndrome and Castleman's Disease. Biology and Management of Unusual Plasma Cell Dyscrasias: Springer; 2017.

6. Karam C, Klein CJ, Dispenzieri A, Dyck PJ, Mandrekar J, D'Souza A, et al. Polyneuropathy improvement following autologous stem cell transplantation for POEMS syndrome. Neurology. 2015; 84: 1981-1987.

7. Dispenzieri A. How I treat POEMS syndrome. Blood. 2012; 119: 5650.
Ann Hematol Oncol - Volume 4 Issue 8 - 2017

ISSN : 2375-7965 | www.austinpublishing group.com

Iftikhar et al. (C) All rights are reserved
Citation: Iftikhar R, Mahmood SK, Satti TM and Qamar un nisa Chaudhry. Poems Syndrome with Vitiligo: Complete Remission and Repigmentation Post Autologous Stem Cell Transplant. Ann Hematol Oncol. 2017; 4(8): 1165. 